\section{Typing renal amyloidosis: immunofluorescence staining can be inconclusive}

The most common nonfamilial forms of amyloidosis that involve the kidney are the $\mathrm{AL}$ (amyloid light chain) and AA (serum amyloid A) types. These two forms require different treatment strategies, and reliable differentiation between types is, therefore, clinically important. Satoskar and co-workers have analyzed 15 renal biopsy samples and used their results to illustrate some of the potential problems in classification of renal amyloidosis.

AL amyloidosis is characterized by deposits that contain monoclonal immunoglobulin light chains; either the $\kappa$ or the $\lambda$ light chain is expected to predominate. The first step in differentiating AA from AL amyloidosis is, therefore, immunofluorescence staining for these two proteins. There were 13 cases of AL amyloidosis in this study; biopsy samples from 11 of these individuals exhibited predominance of either $k$ or $\lambda$ light chains. Four samples showed only moderate-rather than the expected intensestaining of the predominant light chain. All 13 samples were, however, negative when stained for AA. Renal tissue from both cases of $A A$ amyloidosis exhibited strong positive immunostaining for AA but, unexpectedly, light chains were also detected.

The authors conclude that relying solely on immunostaining of light chains could lead to erroneous diagnosis of renal amyloidosis subtype. They recommend staining for AA protein in all cases, except those in which the light chain that is predominant in the amyloid deposits is also detected in both serum and urine. If doubt remains, consultation should be obtained from a center capable of performing more-extensive and more-detailed immunostaining for the other amyloidogenic proteins.

Original article Satoskar AA et al. (2007) Typing of amyloidosis in renal biopsies: diagnostic pitfalls. Arch Pathol Lab Med 131: 917-922

\section{Use of kidneys from donors at high risk for HIV or HCV infection should be considered}

Interpretation by medical practitioners of the transplantation guidelines of the US Centers for Disease Control and Prevention leads most to discard kidneys from potential donors who have behavioral risk factors for HIV or hepatitis $\mathrm{C}$ virus $(\mathrm{HCV})$ infection, even if screening tests for the viruses are negative. These guidelines are, however, now over a decade old, and clinical developments, including the increasing availability of rapid and more-sensitive infection screening, might mean that organs from these increasedrisk donors (IRDs) could safely be used to expand the current pool of donated kidneys. A recent Markov model analysis suggests that kidneys from IRDs who are seronegative following screening for HCV and HIV nucleic acids should be considered for use as allografts.

In comparison with a policy of discarding kidneys from IRDs, a policy of transplanting organs from seronegative (according to both antibody and nucleic-acid testing) IRDs resulted in 20-year improvements in the number of patients who received transplants, waiting time, time with functioning graft, cost of care, and recipient survival, as well as in a greater number of quality-adjusted life-years. Importantly, the discard policy resulted in a higher 20-year incidence of infection overall, attributable to the increased time spent by potential graft recipients on maintenance hemodialysis (which is associated with relatively high risks of acquiring HCV infection). Incidence of HIV infection was, however, higher with the transplant policy than with the discard policy, and the infection rate advantage of using organs from IRDs was lost when nucleic acid screening for HIV and HCV was unavailable.

Original article Schweitzer EJ et al. (2007) Estimated benefits of transplantation of kidneys from donors at increased risk for HIV or hepatitis C infection. Am J Transplant 7: 1515-1525

\section{Weight loss is associated with reduced urinary albumin excretion}

Albuminuria is a well-documented feature of obesity, but the effect of weight change on urinary albumin excretion (UAE) has not been examined in individuals without renal disease. To address this situation, Bello et al. analyzed data from 6,894 members of the general population of Groningen, The Netherlands, who were enrolled in the PREVEND trial.

On the basis of absolute weight change between baseline and a follow-up screening at a mean of 4.2 years, the cohort was divided into three groups: weight loss (>10 kg weight reduction; 